\title{
EFEITO DO FORRAGEAMENTO DE Eciton burchelli (HYMENOPTERA, FORMICIDAE) SOBRE A ARANEOFAUNA DE LITEIRA EM UMA FLORESTA TROPICAL DE TERRA FIRME NA AMAZÔNIA CENTRAL.
}

\author{
Rosamary Silva VIEIRA', Hubert HÖFER ${ }^{2}$
}

\begin{abstract}
RESUMO - Investigamos o efeito do forrageamento de formigas de correição da espécie Eciton burchelli, a qual caça em forma de enxame, sobre a comunidade de aranhas de liteira em uma floresta tropical de terra firme na Amazônia Central. O método usado foi a amostragem de aranhas por triagem manual de liteira, coletando-se 20 quadrados na frente de 7 enxames de caça das formigas e 20 quadrados logo após a passagem das formigas. As aranhas foram identificadas ao nivel de gènero ou espécie e efeitos significativos foram avaliados levando-se em conta o espectro de aranhas-presa destas formigas, conhecido por um estudo na mesma área. Discutem-se as dificuldades e possiveis erros na avaliação do efeito das formigas sobre artrópodos causados pela variância natural de abundâncias e pela restrição da metodologia sob as circunstâncias estudadas.
\end{abstract}

Palavras-chave: Formigas de correição, Ecitonini, aranhas, predação, fauna de liteira.

Effects of the Foraging of Eciton burchelli (Hymenoptera, Formicidae) on the Spider Fauna in the Litter of a Tropical Rain Forest in Central Amazonia.

ABSTRACT - We investigated the impact of swarm-hunting army ants of the species Eciton burchelli on the ground spider assemblage in an Amazonian "terra firme" rain forest. Spiders were collected from 20 quadrat litter samples taken in front of 7 hunting swarms and 20 samples taken in the same areas after army ants had passed, and were identified to genus or species level. Observed significant effects on the abundance of several spider groups are discussed on the base of the prey-spider spectrum of the ants, known from a study in the same forest. We discuss several difficulties and chances of misinterpretation of the results, due to the high naturaI variability of arthropod abundance in the habitat and the restriction of the method under the conditions of the field study.

Key words: Army ants, Ecitonini, spiders, predation, litter fauna.

\section{INTRODUÇÃO}

Eciton burchelli Westwood, 1842 é uma das formigas de correição mais comuns nas regiôes neotropicais, e está inserida num grupo que caracteriza-se pela dieta predatória com atividade de caça em massa, pelo hábito nômade e por alojaremse em ninhos constituídos pelos próprios corpos (Schneirla, 1971). É considerada como uma espécie generalista quando comparada a outras espécies de formigas de correição (Rettenmeyer et al., 1983; Hölldobler \& Wilson, 1990). O forrageamento consiste de massas de operárias guiadas por trilhas químicas e as presas incluem artrópodes de diversos tamanhos (Oster \& Wilson, 1978), predominando adultos e imaturos de outros himenópteros (Hymenoptera), baratas (Blattodea), gafanhotos (Orthoptera), besouros (Coleoptera), aranhas

Instituto Nacional de Pesquisas da Amazônia - INPA, Caixa Postal 478, 69.011-970 Manaus/ AM, Brasil. Endereço atual: Rua Alvorada, 284-ap.204, 69909,380 Rio Branco/AC. e-mail: vieizuma@mdnet.com.br

2 Staatliches Museum für Naturkunde Karlsruhe, Postfach 6209, D-76042 Karlsruhe, Alemanha. 
(Araneae), escorpiōes (Scorpionida) e pseudoescorpiões (Pseudoscorpiones) (Hölldobler \& Wilson, 1990). O impacto causado pela predação destas formigas sobre os artrópodes é considerado pequeno quando comparado ao efeito de outros insetivoros (Franks, 1990).No entanto, deve ser considerada a distinção do efeito das formigas, entre $o$ causado pela simples passagem de um enxame de formigas por uma área (resultando por exemplo em destruicão de teias e fuga de animais) e o efeito da predação de fato. Observa-se que as estimativas podem oferecer margens de erro pela dificil previsão da ação forrageira destas formigas numa área de mata continua, e as constatações divergem conforme $o$ nivel de identificação das presas, bem como as técnicas empregadas na investigação ( Otis et al., 1986; Vieira \& Höfer, 1994; Kaspari, 1996).

Aranhas constituem um dos grupos mais diversos e abundantes na maioria dos ambientes terrestres e em florestas amazônicas representam entre 5 e $10 \%$ dos artrópodes coletados por diversos métodos em estudos ecológicos (Adis \& Schubart, 1984; Adis, 1987). A comunidade de aranhas errantes do solo, apresentando espécies com um modo de vida definitivamente individual deveria representar uma fonte de alimento muito regularmente distribuído em tempo e espaço e assim omni-disponivel para um predador. A relação entre disponibilidade de presas e espectro de presas das formigas de correição foi apresentada por Vieira \& Höfer (1994) e Vieira (1995), mostrando que aranhas constituem uma porção alta do espectro de presas das formigas neste habitat, mas que dentro das aranhas de solo somente poucas espécies são fortemente predadas.

O efeito da predação por formigas de correição sobre populações de aranhas de solo foi considerado baixo por Otis et al. (1986). Entretanto, esta avaliação baseou-se em poucas amostras, e outros autores sustentam que formigas de correição têm grande impacto sobre algumas aranhas. Gasnier et al. (1995) mostraram que alguns grupos de aranhas fogem em massa destas formigas, afetando capturas em armadilhas de tronco.

O objetivo deste trabalho foi o de investigar o efeito das formigas de correição sobre as aranhas como um grupo, e além disto sobre as familias de aranhas (em geral representadas por poucas espécies abundantes), avaliandose as seis familias mais coletadas com amostras de liteira, bem como avaliar e distinguir os processos envolvidos neste efeito.

$\mathrm{Um}$ aspecto mais global do espectro de presas das formigas está sendo tratado em Vieira \& Höfer (1994) e o de tamanho de nicho e sobreposição de nicho de duas espécies de formigas de correição em Vieira (1995).

\section{MATERIAL E MÉTODOS}

O trabalho foi desenvolvido em floresta tropical de terra firme (Reserva " 41 "), inserida nas reservas do Projeto Dinâmica Biológica de Fragmentos Florestais, da cooperação Smithsonian Institution/Instituto Nacional de Pesquisas da Amazônia (INPA), situada aproximadamente $80 \mathrm{~km}$ ao norte de Manaus, com descrição detalhada em Lovejoy (1980) e Lovejoy et al. (1983). 
Para a avaliação do efeito das formigas sobre a população de aranhas foram realizados dois passos:

1. Uma avaliação da alteração provocada pela passagem dos carreiros de E. burchelli sobre as aranhas que ocupam o folhedo foi feita encontrando-se uma colônia de formigas em atividade de caça. $\mathrm{Na}$ "frente de caça" o carreiro era observado para se determinar qual a direção tomada pelas formigas. Distando de 5 a 7 metros desta frente de caça, eram retiradas as amostras de liteira, utilizandose uma sonda de metal de $1 / 16 \mathrm{~m}^{2}$, com 20 sondagens de cada colônia $(\mathrm{N}=7)$. A área tomada pelo enxame foi demarcada por fitas coloridas, onde, imediatamente após a passagem da correição, as amostragens foram repetidas, agora sobre a área que fora forrageada pelas formigas. No mesmo dia da coleta, este material foi examinado retirando-se os organismos manualmente, conservando-os em álcool $70 \%$. As aranhas foram identificadas a nivel de família, gênero e em alguns casos espécies. A partir desta identificação foi feita a comparação da araneofauna de folhedo "antes" e "depois", utilizando-se o teste de chi-quadrado (Siegel, 1975).

2. Foi feita uma comparação dos resultados deste método com observações e dados de estudos paralelos, onde foi investigado o espectro de presas efetivamente predado pelas formigas e a densidade dos grupos mais predados, com estimativa do efeito de predação sobre aranhas-presas do gênero Ctenus, para uma área de aproximadamente $1000 \mathrm{~m}^{2}$ afetada por um enxame de Eciton burchelli durante um dia de caça, descrito em detalhes em Vieira \& Höfer (1994).

\section{RESULTADOS}

Para cada uma das sete colônias de E. burchelli amostradas, foram obtidos 20 pares de amostras de folhiço, de onde foram extraidos 929 artrópodes, 520 antes da passagem das formigas e 409 depois. Limitamo-nos à avaliação de aranhas, sobre as quais concentramos a triagem. Destas, foram coletados 218 indivíduos, identificando-se 23 familias de aranhas (Tab. 1). Foram coletados 130 individuos antes e 88 depois da caçada das formigas, significando um decréscimo de $32 \%$ (Tab. 2). Em coletas de duas colônias encontravamos mais aranhas depois do que antes da passagem das aranhas. Este efeito resulta da grande variação natural na densidade e composição de espécies de aranhas na liteira e do fato que alguns grupos ou espécies de aranhas não foram afetadas pelas formigas (Tab. 1).

Comparando-se as seis familias mais abundantes nas amostras de folhiço, somente Pholcidae, Ochyroceratidae e Ctenidae mostraram decréscimos significativos (Tab. 3). Salticidae e Oonopidae não mostraram diferença significativa e Mygalomorphae, principalmente Dipluridae, mostrou mais individuos depois do que antes da passagem das formigas (Tab. 3).

\section{DISCUSSÃO}

Baseado nas análises nota-se que as formigas de correição podem exercer efeitos diretos ou indiretos sobre algumas familias de aranhas. Verificamos um decréscimo significativo na abundância das familias Pholcidae, Ochyroceratidae e Ctenidae. No entanto, numa avaliação 
Tabela 1. Lista das aranhas coletadas manualmente da liteira em áreas atingidas por 7 colônias de formigas de correição $\left(1.25 \mathrm{~m}^{2}\right.$ coletado antes e depois da passagem).

\begin{tabular}{|c|c|c|c|c|}
\hline FAMILIA & ESPÉCIE & $\mathrm{N}$ (antes) & $N$ (depois) & $\mathrm{N}$ (total) \\
\hline Anapidae & sp. & 2 & 2 & 4 \\
\hline \multirow[t]{4}{*}{ Araneidae } & total & 3 & 4 & 7 \\
\hline & sp. & & & 5 \\
\hline & Alpaida sp & & & 1 \\
\hline & Eriophora sp. & & & 1 \\
\hline \multirow[t]{3}{*}{ Corinnidae } & total & 1 & 3 & 4 \\
\hline & spp. & & & 2 \\
\hline & Corinna sp. & & & 2 \\
\hline \multirow[t]{6}{*}{ Ctenidae } & total & 12 & 4 & 16 \\
\hline & spp. & & & 8 \\
\hline & Ctenus amphora & & & 3 \\
\hline & Ctenus spp. & & & 2 \\
\hline & Ctenus cruisi & & & 2 \\
\hline & Ctenus manauara & & & 1 \\
\hline Deinopidae & Deinopis sp. & 1 & 0 & 1 \\
\hline \multirow[t]{3}{*}{ Dipluridae } & total & 7 & 16 & 23 \\
\hline & sp. & & & 20 \\
\hline & Masteria sp. & & & 3 \\
\hline Heteropodidae & spp. & 8 & 1 & 9 \\
\hline Linyphiidae & spp. & 2 & 3 & 5 \\
\hline Mysmenidae & sp. & 1 & 0 & 1 \\
\hline \multirow[t]{5}{*}{ Ochyroceratidae } & total & 22 & 6 & 28 \\
\hline & Ochyrocera molesta & & & 13 \\
\hline & Ochyrocera n. sp. & & & 8 \\
\hline & n. gen. sp. & & & 5 \\
\hline & sp. & & & 2 \\
\hline \multirow[t]{5}{*}{ Oonopidae } & total & 20 & 20 & 40 \\
\hline & Gamasomorphinae spp. & & & 18 \\
\hline & spp. & & & 14 \\
\hline & Oonopinae spp. & & & 7 \\
\hline & Neoxyphinus sp. & & & 1 \\
\hline \multirow[t]{3}{*}{ Palpimanidae } & total & 1 & 3 & 4 \\
\hline & Fernandezina sp. & & & 3 \\
\hline & sp. & & & 1 \\
\hline \multirow[t]{4}{*}{ Pholcidae } & total & 23 & 7 & 30 \\
\hline & spp. & & & 16 \\
\hline & Micropholcus sp. & & & 12 \\
\hline & Blechroscelis sp. & & & 1 \\
\hline Pisauridae & sp. & 2 & 0 & 2 \\
\hline Prodidomidae & Lygromma gasnieri & 1 & 0 & 1 \\
\hline \multirow[t]{2}{*}{ Salticidae } & total & 13 & 9 & 22 \\
\hline & spp. & & & 22 \\
\hline Segestriidae & $\mathrm{sp}$. & 1 & 2 & 3 \\
\hline Symphytognathidae & Anapistula sp. & 2 & 3 & 5 \\
\hline \multirow[t]{3}{*}{ Theraphosidae } & total & 0 & 2 & 2 \\
\hline & $\mathrm{sp}$ & & & 1 \\
\hline & Cyriocosmus sp. & & & 1 \\
\hline Theridiidae & sp. & 1 & 1 & 2 \\
\hline Theridiosomatidae & sp. & 1 & 1 & 2 \\
\hline Zodariidae & sp. & 2 & 1 & 3 \\
\hline Araneae indet. & & 5 & 0 & 5 \\
\hline total & & & & 218 \\
\hline
\end{tabular}


Tabela 2. Número de aranhas coletadas em liteira antes ( 20 amostras) e depois ( 20 amostras) da passagem dos carreiros de caça de 7 colônias de Eciton burchelli.

\begin{tabular}{lccc}
\hline COLÔNIA & N(ANTES) & N(DEPOIS) & ALTERAÇĀO(\%) \\
\hline 1 & 17 & 2 & -88 \\
2 & 9 & 11 & +18 \\
3 & 14 & 22 & +57 \\
4 & 14 & 11 & -21 \\
5 & 32 & 7 & -78 \\
6 & 21 & 18 & -14 \\
7 & 23 & 17 & -26 \\
\hline TOTAL & 130 & 88 & -32 \\
\hline
\end{tabular}

Tabela 3. Familias dominantes de aranhas: comparação pelo teste Chi-quadrado entre antes e depois do forrageamento de Eciton burchelli (PHOL: Pholcidae, OCHY: Ochyroceratidae, OONO: Oonopidae, MYGAL: Mygalomorphae, SALT: Salticidae, CTEN: Ctenidae; * significante).

\begin{tabular}{lrrrrrr}
\hline FAMILIA & PHOL & MYGAL & OCHY & SALT & OONO & CTEN \\
\hline N antes & 23 & 7 & 22 & 13 & 20 & 12 \\
$N$ depois & 7 & 16 & 6 & 9 & 20 & 4 \\
$X^{2}, 5 \%$ & $8.54^{*}$ & 2.78 & $9.14^{*}$ & 0.72 & - & $4.00^{*}$ \\
$\%$ & -70 & 129 & -73 & -31 & 0 & -67 \\
\hline
\end{tabular}

do espectro de presas de E. burchelli (Vieira \& Höfer, 1994), Pholcidae representou somente $0,6 \%$ dos itens predados por Eciton e Ochyroceratidae não apareceu em nenhuma das amostras de presas utilizadas por esta espécie. A grande maioria das presas observadas foi encontrada numa faixa de comprimento de prosoma de 3 a $6 \mathrm{~mm}$ (Vieira, 1995). Ochyroceratidae são aranhas que em geral medem menos de $3 \mathrm{~mm}$ em comprimento de corpo inteiro, sendo muito poucas as chances de serem predadas.

O efeito negativo das formigas sobre aranhas da família Ctenidae vem reforçar a estimativa feita pelos autores acima, onde Ctenus foi o gênero mais predado por $E$. burchelli ( $84 \%$ das presas).

Estimar abundância de artrópodes de maneira satisfatória é acompanhado de muitas dificuldades, devido á alta variação natural nas densidades ao longo do espaço e do tempo. O esforço na obtenção e na triagem ou extração de amostras suficientes em número e tamanho muitas vezes limita a rigidez dos resultados (um erro padrão alto). É especialmente difícil amostrar diversos grupos de artrópodes com diversos tamanhos, histórias naturais e comportamentos diferentes, utilizandose um só método.

A tarefa se torna ainda mais dificil sob a limitação de tempo disponivel para amostragem e consequentemente a limitação do número de amostras, na situação de amostragem antes e depois da frente de caça das formigas de correição.

$O$ desenho amostral parece adequado para testar a hipótese de que 
certos grupos de artrópodes sejam fortemente afetados pela passagem das formigas de correição em atividade de forrageio, sendo praticamente um experimento controlado de campo, mas a nossa experiência ganha em trabalhos paralelos mostrou que poderiam surgir resultados enganadores deste estudo, Um grupo que não apareceu como presa nas coletas de espectro de presas de Eciton burchelli (Ochyroceratidae, Vieira \& Höfer, 1994) apresenta efeito significativo neste estudo. Estas aranhas são as mais abundantes amostradas com o método de quadrados do tamanho usado e assim a chance de uma diferença significativa antes/depois é alta, Obviamente não podemos descartar a possibilidade do efeito ser resultado de influência indireta do forrageio destas formigas, sendo a predação por outros predadores oportunistas ou o escondimento ou a fuga.

Em outros estudos considerou-se significativo o efeito de forrageamento das formigas de correição sobre Pseudoescorpiões, e a falta de efeito significativo sobre aranhas como grupo (Otis et al., 1986). No entanto, o presente estudo mostra que com a mesma técnica, mas analisando as aranhas em outro nivel taxonômico, este efeito mostra um quadro diferente.

$\mathrm{Na}$ frente de caça, muitas aranhas foram observadas subindo pelos troncos, e algumas rapidamente foram capturadas por pássaros, principalmente dendrocolaptídeos. Outros destinos também são observados, como predação por outros pássaros que também se incluem entre o grupo de seguidores (Willis \& Oniki, 1988; Harper, 1989; Willis, 1992).
Outros predadores, como lagartos e macacos, são citados como seguidores (Willis \& Oniki, 1988; Rylands et al., 1989), assim como possiveis agentes de parasitismo, como dipteros Tachinidae. No entanto, estes grupos năo foram observados e/ou quantificados, acompanhando a falta de avaliação sobre a possibilidade de fuga que as aranhas podem experimentar.

Desta forma, é certo que a passagem dos carreiros de E. burchelli provoca uma mudança na estrutura espacial da comunidade da liteira, influenciando mesmo as aranhas que não sâo efetivamente predadas por estas formigas, expulsando-as de seus sítios originais. As passagens das formigas aumentam o risco de predação das aranhas, por aumentarem a exposição destes organismos a outros predadores e parasitas. As aranhas removidas ainda terão um gasto energético para conseguirem voltar às moradias, ou procurar outros lugares para se instalarem.

\section{AGRADECIMENTOS}

Os agradecimentos são prestados à CAPES, Smithsonian Institution e a DFG (Conselho Alemão de Pesquisa) pelo suporte financeiro, aos revisores pelas criticas e ao Sr. Antonio Cardoso pela preciosa ajuda nos trabalhos de campo.

\section{Bibliografia citada}

Adis, J. 1987. Extraction of arthropods from neotropical soils with a modified Kempson apparatus. J. Trop. Ecol., 3:131-138.

Adis, J.; Schubart, H. O. R. 1984. Ecological research on arthropods in Central Amazonian forest ecosystem with recommendations for study procedures. In: J.H. Cooley \& F.B. Golley (eds.). Trends in ecological research for the 1980. NATO 
Conference Series, I: Ecology, Plenum Press, New York, London, p11-144.

Franks, N.R. 1990. Ecologia y regulacion de poblacion de la hormiga guerrera Eciton burchelli. In: E.G. Leigh Jr; A.S. Rand; D.M. Windsor (eds.). Ecologia de un Bosque Tropical: Ciclos Estacionales y Cambios a Largo Plazo. Smithsonian Tropical Research I., Balboa, Panamá, p453-460.

Gasnier, T.; Höfer, H.; Brescovit, A.D. 1995. Factors affecting the "activity-density" of spiders on tree trunks in an Amazonian rainforest. Ecotropica, 1(2):69-77.

Harper, L.H. 1989. The persistence of ant-following birds in small Amazonian forest fragments. Acta Amazonica, 19;249-263.

Hölldobler, B.; Wilson, E.0. 1990. The Ants Cambridge, Mass., Belknap Ed.

Kaspari, M. 1996. Litter ant patchiness at the $1-\mathrm{m}^{2}$ scale: disturbance dynamics in three Neotropical forests. Oecologia, 107:265-273

Lovejoy, T.E. 1980. Discontinuous wilderness: minimum areas for conservation. Parks, $5(2): 13-15$.

Lovejoy, T.E.; Bierregard, R.O.; Rankin, J.M.; Schubart, H.O.R, 1983. Ecological dynamics of forest fragments. In: S.L. Sutton, T.C. Whitmore; A.C. Chadwick (eds.). Tropical Rainforest. Ecology and Management. Blackwell, Scient. Publ., Oxford, p377-385.

Otis, G. W.; Santana, C.E.; Crawford, D.L.; Higgins, M.L. 1986. The effect of foraging army ants on leaf-litter arthropods. Biotropica, I8(1):56-61.

Oster, G.F.; Wilson, E.0. 1978. Social InsectsCaste and Ecology. New Jersey, Princeton Univ. Ed.

Rettenmeyer, C.W. 1963. Behavioral studies of army ants. The University of Kansas Science Bulletin, 44(9):281-465.
Rettenmeyer, C. W.; Chadab-Crepet, R.; Naumann, M.G.; Morales, L. 1983. Comparative foraging by neotropical army ants. In: P. Jaisson (ed.). Social insects in the tropies, Vol.2. Proc. 1st International Symposium IUSSI and Sociedad Mexicana de Entomologia, Cocoyoc, Morelos, México 1980. Universite Paris-Nord, Paris, p 59-73.

Rylands, A.B.; Cruz, M.A.O.M.\& Ferrari, S.F. 1989. An association between marmosets and army ants in Brazil. Journal of Tropical Ecology, 5:113-116.

Schneirla, T.C. 1971. In: H.R. Topoff (ed.). Army Ants; a Study in Social Organization. Freeman, San Francisco.

Siegel, S, 1975. Estatistica não paramétrica (para as ciências do comportamento). New York, McGraw-Hill.

Vieira, R.S. 1995. Efeito de forrageamento de formigas de correição (Hym.: Formicidae-Ecitonini) sobre a comunidade de aranhas (Araneae) em uma floresta tropical de terra firme na Amazônia Central. Dissertação de Mestrado. INPA-FUA, Manaus.

Vieira, R.S.; Höfer, H. 1994. Prey spectrum of two army ant species in central Amazonia, with special attention on their effect on spider populations. Andrias, 13:189-198.

Willis, E.O.; Oniki, Y. 1988. Na trilha das formigas carnivoras. Ciência Hoje, 47(8):26-32.

Willis, E.O. 1992. Behavior and ecology of barred woodcreepers, Dendrocolaptes certhia (Aves, Dendrocolaptidae). Boletim Museu Paraense Emilio Goeldi Zoologia, 8(1):151-216 